\title{
3D Reconstruction of Simulated Vapor Cloud Explosion Triggered by Major Hazard Installation
}

\author{
Wenjiang Chen, Yan Yong, Zhaoji Hu, and Hongbo Su
}

\begin{abstract}
As one of the most frequently occurring accidents in a petrochemical enterprise, a vapor cloud explosion (VCE) is likely to occur when the vapor cloud is formed due to the leakage of a major hazard installation (MHI) storing massive flammable and explosive media. In particular, a VCE in the storage area of petrochemical enterprise may cause domino accidents if it is out of control. Considering the safety and economy of large-scale accident experiment, this paper proposes a three-dimensional (3D) reconstruction method to describe VCE triggered by MHI. The method describes 3D hazard characteristics of VCE based on the Netherlands Organization (TNO) multi-energy method. To enhance the sensing cognition of VCE, a $3 D$ reconstruction system is developed based on the virtual reality (VR) idea. Finally, the system is applied in an actual petrochemical enterprise to simulate the dynamic effects and the consequences of VCE. The results show that the $3 \mathrm{D}$ reconstruction system is of lifelike and visual characteristics, which not only embodies the great practical application value of the VR technology in the field of safety engineering, but also provides references for safety management, disaster prediction, risk assessment, emergency response and safety planning of petrochemical enterprises.
\end{abstract}

Index Terms-Vapor cloud explosion, major hazard installation, virtual reality technology, three-dimensional reconstruction.

\section{INTRODUCTION}

Safety is an important prerequisite for ensuring human survival and quality of life. A large number of chemical industrial parks have been built with the rapid development of industries. The number of hazard installations that store large quantities of hazardous substances increases as well. Once occurrence of an accident, it usually causes massive casualties and huge economic loss. It is a great challenge for safety management of hazard installations especially major hazard installations (MHI) in chemical industrial parks.

In a view of safety management of MHI, a lot of research work has been done [1]-[4]. Due to the characteristics of MHI

Manuscript received August 10, 2018; revised October 18, 2018.

This work was supported in part by Jiangxi Provincial Department of Education, China, Grant No. GJJ160083.

W. Chen and Z. Hu are with the Nanchang University, Nanchang 330031, China (e-mail: chenwenjiang@ncu.edu.cn, zhjhu2005@163.com).

W. Chen, Y. Yong and H. Su are with the Florida Atlantic University, FL 33431, USA (e-mail: chenw@fau.edu, yongy@fau.edu, suh@fau.edu) accidents and the limitation of various objective factors, it is impossible for people to analyze the consequences of accidents by the means of the real experiments. With the rapid development of computer technologies, computer simulations make up for these defects, such as the widely used commercial software packages named DNV SAFETI [5] and PHAST [6]. These simulators can perfectly reflect the two-dimensional(2D) level information of accident consequences. However, the numerous accident cases, including leakage, diffusion, fire and explosion, show that consequences are often of three-dimensional (3D) characteristics. In the aspect of 3D simulation of accidents, there are als o some software packages, such as the simulators named Kemeleon FireEx [7] and FLACS [8] based on computational fluid dynamics (CFD). Although these simulators can accurately describe the 3D characteristics of accidents, the efficiencies are very low so that the professionals will take much time to obtain the analysis results. With the wide application of virtual reality (VR), geographic information system (GIS) and 3D visualization technologies in the fields such as military [9], medical [10], [11], architecture [12], [13] and other engineering fields [14], [15], these technologies have been gradually introduced and applied in the field of safety engineering. However, these simulators didn't realize the 3D reconstruction of major accidents in chemical industrial parks, especially in terms of 3D effects including occurrence, development and consequence simulation of MHI accidents. As is known to all, explosion that is one of the most destructive accidents in the petrochemical industry usually happens in a flash. Vapor cloud explosion (VCE) is one of the most typical explosions. If an original VCE is reconstructed by computer technologies, it will be much more meaningful for safety management and accident prevention of MHI.

In order to address the limitations above, we developed a tool called 3DRS_VCE (three-dimensional reconstruction system of vapor cloud explosion) in this work. The contributions of 3DRS_VCE are as follows. First, 3DRS_VCE can establish the virtual environment (VE) of VCE to reconstruct the accident scene that contains the surrounding geographic information. It is a flexible tool for managers to supervise the enterprise, especially hazard installations. Second, 3DRS_VCE is designed to perform the consequence evaluation of VCE, which can analyze and predict the evolution trend of consequences. Third, 3DRS_VCE can also vividly display the analysis results in the VE by human-computer interaction. It can provide technical support for supervision of hazard installations, disaster prevention, emergency response and safety planning of petrochemical 
enterprises, etc.

The organizational structures of this paper are as follows. In section II, we introduce the methods of quantitative consequence assessment which is used to describe 3D characteristics of VCE, and the development methods of 3DRS_VCEto help understand the design and implementation processes. In section III, 3DRS_VCE is applied in an actual petrochemical enterprise to demonstrate its workflow and functions. Then we discuss the application results in section IV. Finally, the application prospects of 3DRS_VCE and the future research are presented in section $\mathrm{V}$.

\section{METHODOLOGIES}

\section{A. 3D Characteristic Analysis of VCE}

According to the mechanism of accident, the explosive vapor cloud is easily formed due to the flammable and explosive substances mixing with the air. In case of an ignition source in it, there will be a great possibility of VCE, accompanying with blast wave overpressure. As the blast wave expands in the surrounding space, VCE is of the evident 3D characteristics. In order to scientifically and vividly describe the 3D consequences of VCE, the 3D geometry of VCE and calculation methods of the characteristic parameters are established as follows.

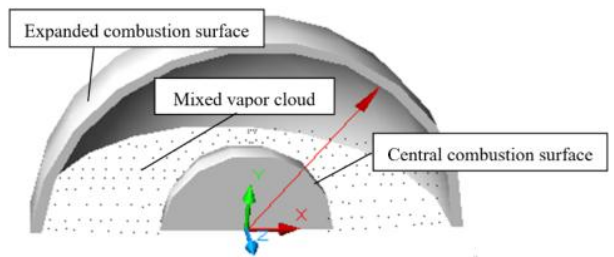

Fig. 1. The geometric model of VCE.

Fig. 1 shows the geometric shape of VCE. The process of VCE occurrence can be described as follows.

Once occurrence of leakage from hazard installations storing flammable and explosive substances, the vapor cloud is formed by mixing with the surrounding air. When the vapor cloud is ignited by a fire source, a spherical combustion surface occurs as the center of combustion. And then the combustion surface expands towards the surrounding space. With the expansion of combustion surface, the spherical combustion surface gets much bigger and the surrounding vapor cloud is ignited further. In this case, the extent of combustion gets much stronger and the blast wave is formed due to the rapid speed of expansion.

At present the Netherlands Organization (TNO) multi-energy method is a common and classic algorithm of simulating and predicting VCE. When the method is used by selecting an appropriate intensity of explosion, the scaled peak overpressure and dimensionless positive phase duration can be obtained from the characteristic curves of blast wave, as shown in Fig. 2 [16]. Fig. 2(a) shows the relationship between the scaled peak overpressure and the dimensionless distance, and Fig. 2(b) shows the relationship between the dimensionless positive phase duration and the dimensionless distance. Each curve corres ponds to an intensity of explosion source. And the intensity is usually equal to 7 for a certain limited space similar to the storage tank area in chemical industrial park [17].

The damage extent of VCE depends on the characteristic parameters including the peak overpressure of blast wave and the positive phase duration of blast wave. The method of calculating characteristic parameters is established by the least square method based on the characteristic curve (corresponding to No. 7 shown in Fig. 2). The peak overpressure of blast wave, $P_{s}(\mathrm{~Pa})$, can be calculated by [17]

$$
P_{s}=P_{a} \exp \left(-0.9126-1.5058 \ln \left(r^{\prime}\right)+0.1675 \ln ^{2}\left(r^{\prime}\right)-0.0320 \ln ^{3}\left(r^{\prime}\right)\right)(1)
$$

where $P_{a}(\mathrm{~Pa})$ is the air pressure, and $r$ ' is the dimensionless distance calculated by

$$
r^{\prime}=X /\left(E / P_{a}\right)^{1 / 3}
$$

where $X(\mathrm{~m})$ is the distance from object to the center of explosion source, and $E(J)$ is the energy of explosion calculated by

$$
E=1.8 \eta m_{e} \Delta H_{c}
$$

where $\eta$ is the equivalent coefficient of vapor cloud which usually equals $0.04, m_{e}(\mathrm{~kg})$ is the quality of fuel involved in explosion, and $\Delta H_{c}(\mathrm{~J} / \mathrm{kg})$ is the combustion heat value of fuel. The positive phase duration of blast wave, $T$ (s), can be calculated by [18]

$$
T=E^{1 / 3} \exp \left(-1.2500+0.5038 \ln \left(r^{\prime}\right)-0.1118 \ln ^{2}\left(r^{\prime}\right)\right)
$$

In addition, the radius of death, $R_{l}(\mathrm{~m})$, is calculated by Eq. (5) as a result of the head affected by blast wave. The radius of serious injury, $R_{2}(\mathrm{~m})$, is calculated by Eq. (6) corresponding to $50 \%$ of ear drum membrane broken by blast wave. The radius of slight injury, $R_{3}(\mathrm{~m})$, is calculated by Eq. (7) corresponding to $1 \%$ of ear drum membrane broken by blast wave [17], [18].

$$
\begin{aligned}
& R_{1}=1.98\left(E / Q_{p}\right)^{0.447} \\
& R_{2}=9.187\left(E / Q_{p}\right)^{1 / 3} \\
& R_{3}=17.877\left(E / Q_{p}\right)^{1 / 3}
\end{aligned}
$$

where $Q_{p}$ is the combustion heat value of propane.

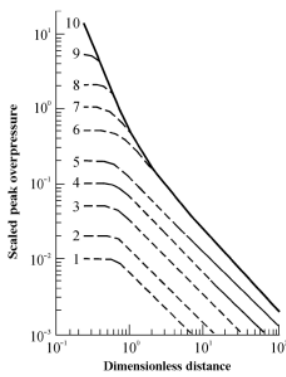

(a)

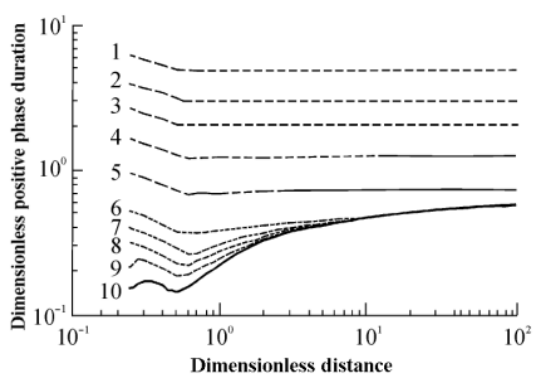

(b)
Fig. 2. The characteristic curves of blast wave.

\section{B. 3D Reconstruction Method of VCE}

To efficiently calculate the characteristic parameters and 
vividly describe the consequences of VCE, a 3D reconstruction system of simulated VCE named 3DRS_VCE is developed in this paper. The development process and implementation methods are as follows.

Based on the 3D characteris tic analys is methods of VCE, the technical proposal of 3DRS_VCE is designed in Fig. 3. According to the characteristics of distributed MHI, there are other geographic information except MHI. The geographic information affects the occurrence of accident and its evolution. And the accidents can also affect the surrounding environment. To objectively describe the consequences of accidents, the surrounding geographic information must be considered in the process of consequence analysis. Therefore, it is neces sary to reconstruct a 3D scene of accidents. And the 3D geographic information can be stored in the database as a map.

Based on the analys is methods of characteristic parameters, the consequences can be analyzed in the reconstructed virtual scene by combining the initial data of hazard installations. To improve the efficiency of calculation, a module of consequence assessment is designed as well.

To visually describe the results of assessment, 3D effects including occurrence of VCE and its influence ranges are shown in the virtual scene. The different influence ranges corresponding to different extents of damage can be analyzed and displayed in a form of 3D effects to meet users' needs.

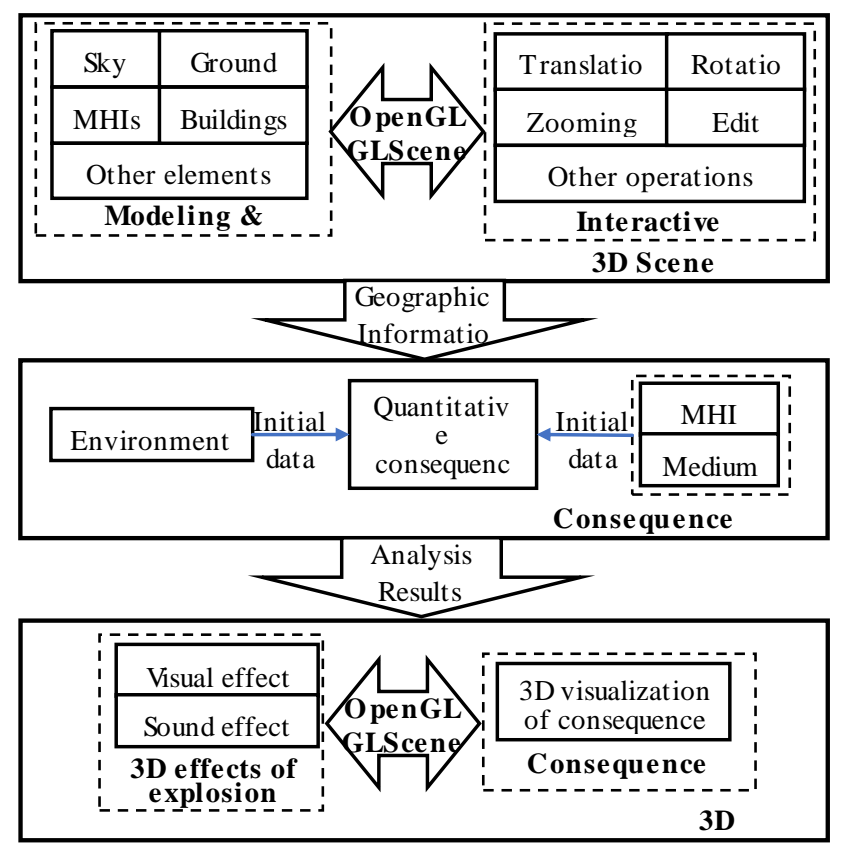

Fig. 3. The technical proposal of system.

This system is developed by Delphi as the main programming tool to improve the efficiency of designing human-computer interaction interfaces, and SQL Server as the database. OpenGL is a 3D graphics library with strong independence. Its advantages include flexible operation, real-time control and transplanting cross platform. OpenGL is a perfect tool considering interactive modeling, expansibility, cross-platform and implementation level, etc. However, OpenGL is insufficient for complex 3D scene so that it takes much time to program tedious code of entity modeling.
GLScene, which is a component package of OpenGL, provides the visual design components so that the complexity of OpenGL can be simplified, and flexibly reconstructs 3D scene by combining with Delphi. Thus, OpenGL/GLScene is introduced as the 3D development tool in this work, as shown in Fig. 3. By using OpenGL/GLScene, the following sections describe the implementation methods of the main modules in the process of simulating VCE, such as 3D virtual scene, 3D effects of explosion and 3D visualization of consequences.

Reconstructing a 3D virtual scene is actually the process of $3 \mathrm{D}$ entity modeling and rendering. The 3D geometric model of object is rendered in the $3 \mathrm{D}$ coordinates. By a series of transformations and processing, 3D coordinates can be then transformed to the pixel positions on the computer screen, so that the corresponding 3D graphics can be drawn and displayed. Based on OpenGLGLScene, the process of reconstructing $3 \mathrm{D}$ virtual scene is described as follows.

- Scene layout by setting light source and viewpoint.

- With the prerequisite of programming sky and ground, 3D entity modeling including MHI and other objects.

- Rendering the established geographic elements and virtual environment by setting colors and textures.

- Displaying the 3D virtual scene on the 2D screen by transformations including projection and viewport.

The process is modularized in this system to provide users with a convenient function of reconstructing arbitrary 3D virtual scenes.

VCE is usually a violent burning phenomenon that occurs instantaneously. OpenGL/GLScene animation, which is based on VR idea including visualizing the abstract objects, embedding sound and visual effects, is introduced in th is work to simulate the dynamic effects of VCE occurrence. The main steps are as follows.

- Defining a picture material library.

- Saving the preprocessed pictures in the library.

- Playing these pictures rapidly based on the principle of mankind vision.

The process of implementation is shown in Fig. 4, where visual effect is achieved by programming and invoking the unit file of explosion effect, and sound effect is achieved by creating a library of loading sound source and a class of playing sound source. Then the visual and sound effects are played synchronously by setting an animation metronome which is a switch controlling accident simulation.

According to the Eq. (5)-Eq. (7), the casualty radii, such as death, serious injury and slight injury, are efficiently analyzed in the module of consequence assessment. In this paper, the casualty radii are drawn and displayed to enhance the sensing cognition of accident severity. The implementation process of drawing casualty radii is described as shown in Fig. 5, where analys is result $R_{i}$ is transmitted as the initial value of $R$ which compares with tank radius $R_{t}$ recalled from the database. The comparis on result decides whether the list of affected objects is firstly output. Then the casualty radius is drawn and displayed by defining an object class named TGLSphere and setting its properties including color, slices, stacks and radius. The list of affected objects shows the buildings and tanks which are within the influence range. 


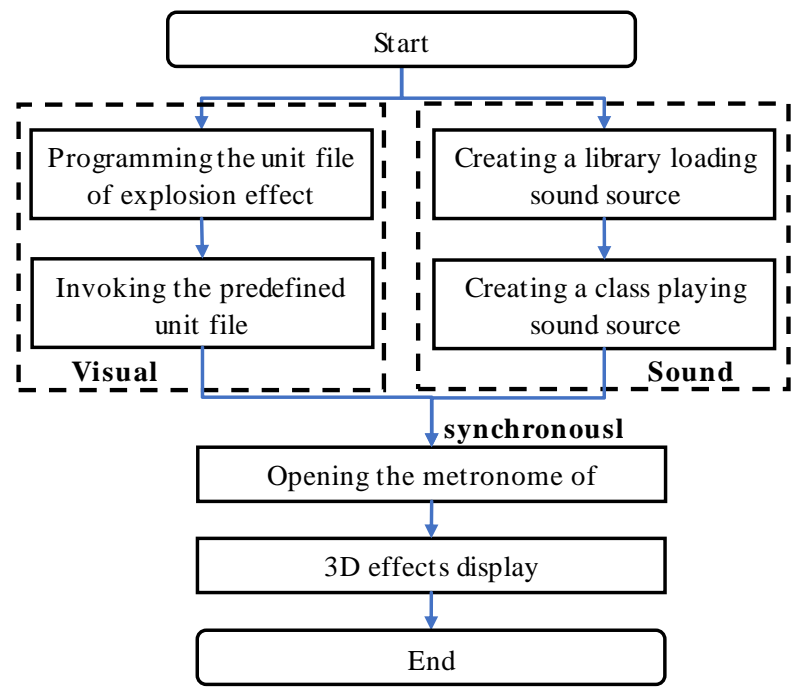

Fig. 4. The implementation method of 3D visualization of VCE.

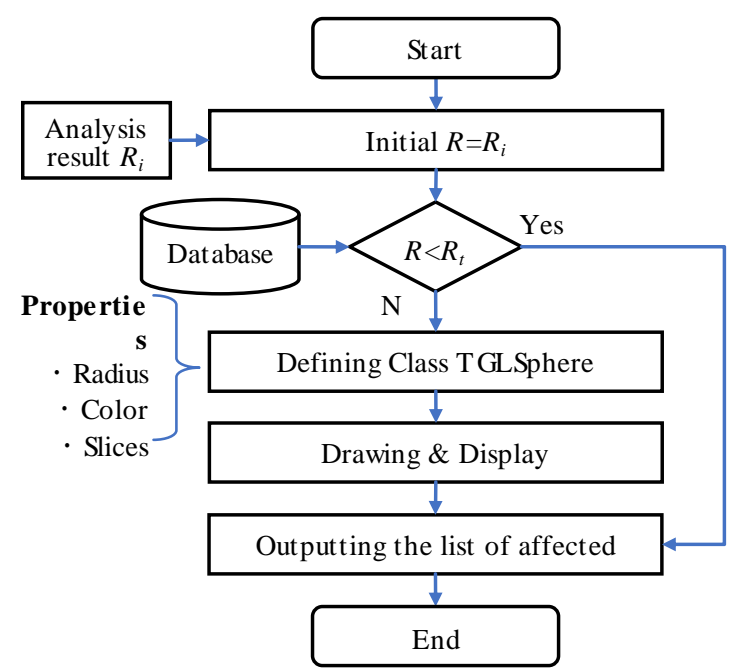

Fig. 5. The process of drawing casualty radius of VCE.

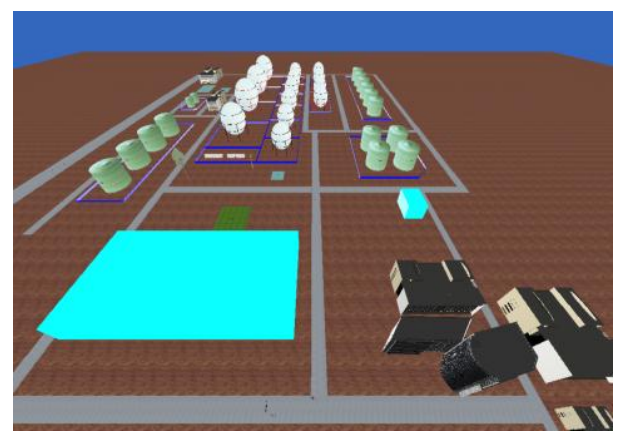

Fig. 6. 3D reconstruction scene.

\section{RESULT}

In accordance with the above methodologies, 3DRS_VCE was developed and applied in an actual petrochemical enterprise. The following sections introduce the details of simulating an accident of VCE triggered by MHI to demonstrate the workflow and functions of 3DRS_VCE.

\section{A. Case Profiles}

There are five propane tanks with a volume of $3500 \mathrm{~m}^{3}$, five propane tanks with a volume of $3000 \mathrm{~m}^{3}$, three butane tanks with a volume of $10000 \mathrm{~m}^{3}$, one butane tank with a volume of $6500 \mathrm{~m}^{3}$ and two horizontal tanks with a volume of $100 \mathrm{~m}^{3}$ at the storage area of the enterprise. The natural conditions of the city in which the enterprise operates are as follows: annual mean wind speed equals $2.4 \mathrm{~m} / \mathrm{s}$, annual mean temperature equals $21.9^{\circ} \mathrm{C}$ and annual mean relative humidity equals $81 \%$.

\section{B. Simulation Results}

(1) $3 \mathrm{D}$ reconstruction of accident scene

Based on the process of 3D entity modeling and rendering in section II, a virtual scene of VCE is reconstructed by utilizing OpenGL/GLScene, as shown in Fig. 6. 3DRS_VCE eventually simulates the geographic elements corresponding to the actual scene, such as MHI (including vertical tank, horizontal tank and spherical tank), buildings, green area, water area, road and so on. The efficiency of establishing 3D scene is greatly high to meet the needs of reconstructing the arbitrary accident scene in petrochemical enterprise because each geographic element is modularized.

(2) Consequence assessment and analys is

In the reconstructed accident scene shown in Fig. 6, 3DRS_VCE can quickly calculated and analyzed the consequences of VCE by Eq. (1)-Eq. (7) after setting variable parameters by the initial data from accident scene. The analysis results as shown in Fig. 7, including the peak overpressure of blast wave, duration of blast wave, radius of death, radius of serious injury and radius of slight injury, are shared with the module of 3D simulation. In addition, 3DRS_VCE can also estimate the number of casualties and property losses caused by VCE if setting the densities of population and property.

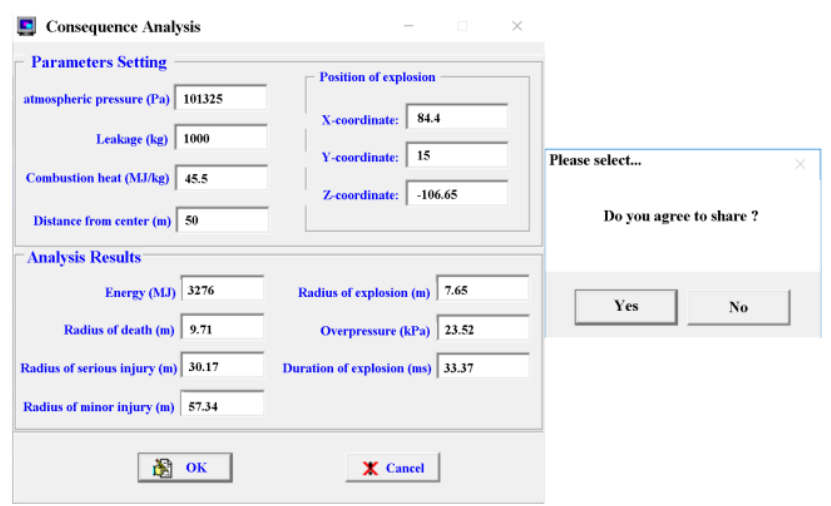

Fig. 7. Parameters setting and consequence analysis.

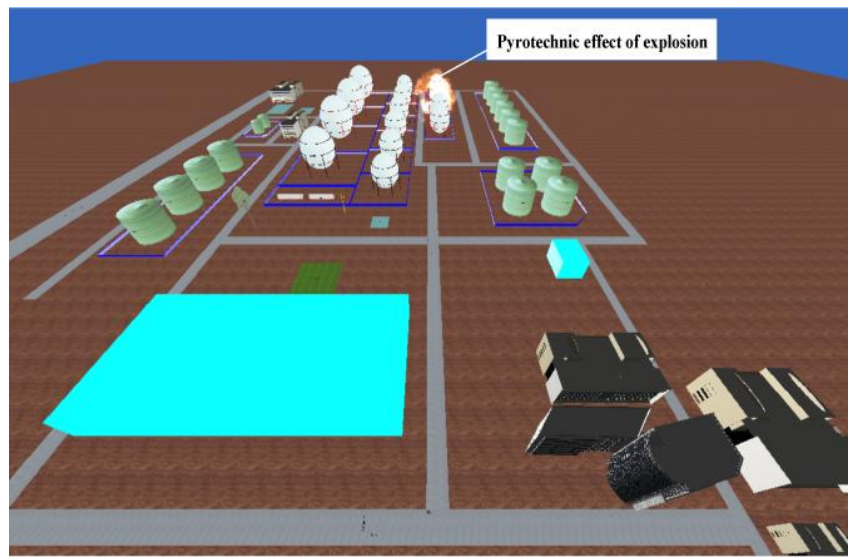

Fig. 8. 3D effect of explosion. 


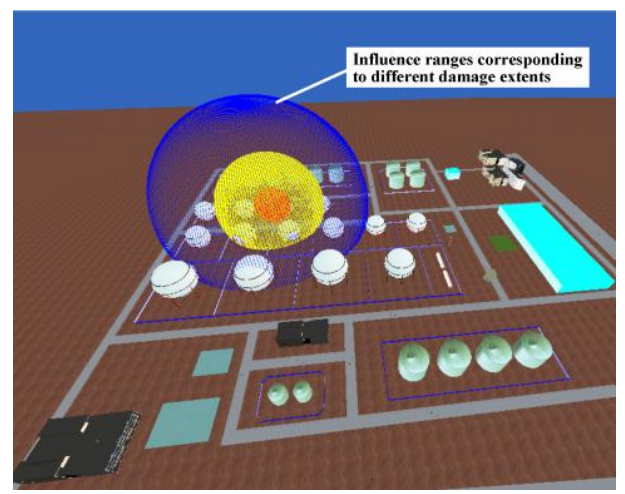

Fig. 9. 3D visualization of different influence ranges.

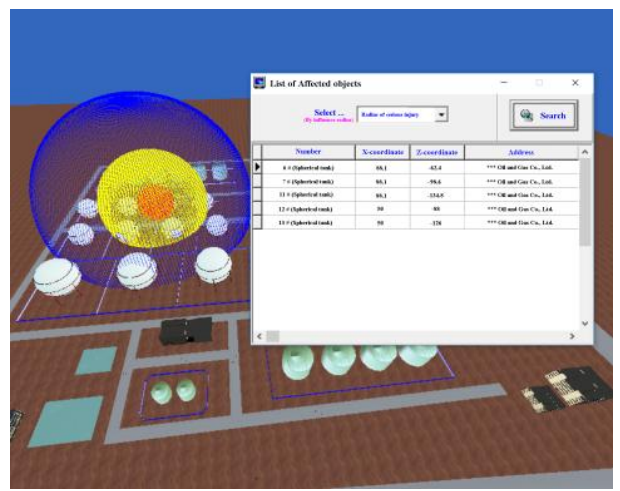

Fig.10. The list of affected objects.

(3) 3D visualization simulation

Based on the method shown in Fig. 4, 3D effects of explosion are achieved as shown in Fig. 8. Based on the analysis results shown in Fig. 7, influence ranges, corresponding to different damage extents including death, serious injury and slight injury, are drawn and displayed with different colors in Fig. 9 by the method shown in Fig. 5. Finally, the lists of affected objects within different influence ranges, as shown in Fig. 10, can als o be output by the method shown in Fig.5.

\section{DISCUSSION}

\section{A. Analysis of Simulation Results}

Based on the methods in section II, the overpressure and duration of blast wave can be efficiently calculated by 3DRS_VCE in this work. The relationship between overpressure and distance is described as shown in Fig. 11, and the relationship of duration and distance is described as shown in Fig. 12. The simulation results show that the trends of the relationships are the same as the characteristic curves proposed by TNO in Fig. 2.

\section{B. Comparison of Simulation effects}

From the simulation results, 3DRS_VCE is of 3D immersive sense and high efficiency besides the reliability of computational model.

(1) 3D immersive sense

Compared with the traditional 2D simulators of accidents introduced in section $\mathrm{I}$, the $3 \mathrm{D}$ reconstruction system embodies the superiorities including much more perfectly describing the $3 \mathrm{D}$ characteristics of accidents and more vividly displaying the consequences in the virtual accident scene, as shown in Fig. 6, Fig. 8- Fig. 10. The 3D reconstruction system can also achieve the 3D visualization functions including the accident occurrence, animation effects and dynamic consequences in the virtual scene, to enhance the sensing cognition of VCE.

(2) High efficiency

Compared with other 3D simulators based on CFD, the calculation efficiency of $3 \mathrm{D}$ reconstruction system is much higher, as shown in Fig. 7. Because the 3D reconstruction system is developed based on TNO method that is one of classic algorithms. It is well known that CFD is based on a grid for the analys is of accidents, so that the profes sionals will take much more time to obtain the analysis results [8], [19]. The high efficiency of consequence as sessment is significant for accident prevention and prediction, and taking emergency measures to stop from the domino effect of accidents.

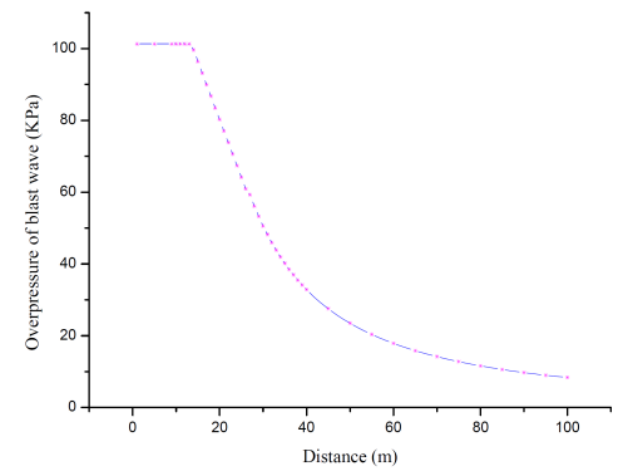

Fig. 11. The relationship between overpressure and distance.

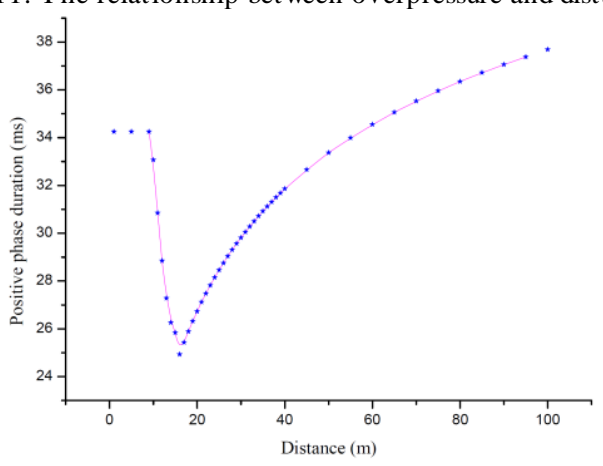

Fig. 12. The relationship between duration and distance.

\section{SUMMARY AND FUTURE WORKS}

The results of simulation application show that 3DRS_VCE can vividly describe the 3D characteristics of VCE. It shows that the $3 \mathrm{D}$ characteristic analysis method of VCE is reasonable, and the $3 \mathrm{D}$ simulation system that is developed based on the 3D reconstruction method is feasible and reliable to simulate the actual geographic information, the process of accident occurrence, dynamic effects and consequences visualization. The modularized $3 \mathrm{D}$ reconstruction method proposed in this paper can not only simulate the major accidents including explosion in petrochemical enterprises, but also simulate large-scale accidents or disasters in other fields.

In the future research, we will introduce internet of things and artificial intelligence technologies into this tool to 
establish an intelligent platform for real-time monitoring and risk early warning of major accidents, predicting the evolution trends of consequences to prevent the domino effect of accidents. The platform will provide decision support for disaster prevention, emergency response and safety planning of industrial and urban areas.

\section{ACKNOWLEDGMENT}

This work was supported by Jiangxi Provincial Department of Education, China, Grant No. GJJ160083.

\section{REFERENCES}

[1] D. Lisbona, J. Januszewski, H. Balmforth, and M. Wardman, "Societal risk assessment of major hazard installations using QuickRisk," Process Safety and Environmental Protection, vol. 89, no. 6, pp. 404-414, November 2011.

[2] B. Woodcock and Z. Au, "Human factors issues in the management of emergency response at high hazard installations," Journal of Loss Prevention in the Process Industries, vol. 26, no. 3, pp. 547-557, May 2013.

[3] V. Casson Moreno, and V. Cozzani, "Major accident hazard in bioenergy production," Journal of Loss Prevention in the Process Industries, vol. 35, pp. 135-144, May 2015.

[4] M. Zhao, and X. Liu, "Regional risk assessment for urban major hazards based on GIS geoprocessing to improve public safety," Safety Science, vol. 87, pp. 18-24, August 2016.

[5] M. Spoelstra, S. Mahesh, E. Kooi, and P. Heezen, "Domino effects at LPG and propane storage sites in the Netherlands," Reliability Engineering and System Safety, vol. 143, pp. 85-90, November 2015.

[6] H. W. M. Witlox, M. Harper, S. Natarajan, and A. Williamson, "Modelling of time-varying dispersion for elevated pressurised releases without rainout," Journal of Loss Prevention in the Process Industries, vol. 35, 283-292, May 2015.

[7] C. B. Jang, and S. W. Choi, "Simulation and damage analysis of an accidental jet fire in a high-pressure compressed pump shelter," Safety Health at Work, vol. 8, no. 1, pp. 42-48, March 2017.

[8] A. Borg, B. Paulsen Husted, and O. Njå, "The concept of validation of numerical models for consequence analysis," Reliability Engineering and System Safety, vol. 125, pp. 36-45, May 2014.

[9] Z. Zhu, Q. Dong, J. Feng, and A. Liu, "Research on visula simulation technology for air-to-air missile based on glscene," Journal of Projectiles, Rockets, Missiles and Guidance, vol. 28, no. 5, pp. 74-76, 82, October 2008.

[10] B. Zhang, X. Wang, X. Liang, and J. Zheng, "3D reconstruction of human bones based on dictionary learning," Medical Engineering and Physics, vol. 49, pp. 163-170, November 2017.

[11] J. L. Cercos-Pita, I. R. Cal, D. Duque, and G. Sanjuán de Moreta, "NASAL-Geom, a free upper respiratory tract 3D model reconstruction software," Computer Physics Communications, vol. 223, pp. 55-68, February 2018.

[12] N. Lercari, "3D visualization and reflexive archaeology: A virtual reconstruction of Çatalhöyük history houses," Digital Applications in Archaeology and Cultural Heritage, vol. 6, pp. 10-17, September 2017.

[13] H. Kim and H. Kim, "3D reconstruction of a concrete mixer truck for training object detectors," Automation in Construction, vol. 88, pp. 23-30, April 2018.

[14] F. Bergamasco, A. Torsello, M. Sclavo, F. Barbariol, and A. Benetazzo, "WASS: An open-source pipeline for 3D stereo reconstruction of ocean waves," Computers \& Geosciences, vol. 107, pp. 28-36, October 2017.

[15] P. Hu, T. Komura, D. Li, G. Wu, and Y. Zhong, "3D High-quality textile reconstruction with synthesized texture," Procedia Computer Science, vol. 108C, pp. 355-364, June 2017.
[16] H. Ozog, G. A. Melhem, and A. D. Little, "Facility siting - Case study demonstrating benefit of analyzing blast dynamics," in Proc. International Conference and Workshop on Process Safety Management and Inherently Safer Processes, AIChE/CCPS, October 1996, pp. 293-315.

[17] R. Zhang, G. Chen, H. Zhang, and W. Yan, "Engineering Applications of TNO Multi-Energy Method in VCE Simulation Assessment," Journal of South China University of Technology (Natural Sicence Edition), vol. 34, no. 5, pp. 109-114, May 2006.

[18] W. Chen, "Research on 3D dynamic visualization consequence simulation of MHI's fire and explosion accidents," M.S. thesis, South China University of Technology, Guangzhou, China, 2010.

[19] M. M. Jujuly, A. Rahman, S. Ahmed, and F. Khan, "LNG pool fire simulation for domino effect analysis," Reliability Engineering and System Safety, vol. 143, pp. 19-29, November 2015.

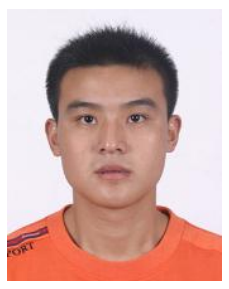

Wenjiang Chen received a B.Eng. degree in Safety Engineering from North China Institute of Science and Technology, China, in 2007, and M.S. degree in Safety Technology and Engineering from South China University of Technology, China, in 2010 , respectively. He is currently pursuing the $\mathrm{Ph} . \mathrm{D}$. degree. He is currently a visiting scholar at Florida Atlantic University, USA. His research interests include safety management information system, accident modeling, and consequence simulation based on computer technologies.

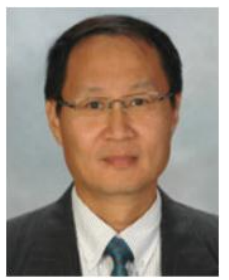

Yan Yong is currently department chair and professor with Florida Atlantic University, Boca Raton, FL, USA. His research interests include random vibrations, structural dynamics, earthquake engineering and turbulent flows. He received a Ph.D. degree in Aeronautical and Astronautical Engineering from University of Illinois at Urbana-Champaign, USA, in 1987.

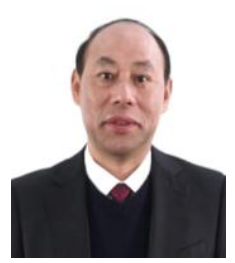

Zhaoji Hu is currently a professor with Nanchang University, China. His research interests include development of new process equipment, failure analysis and accident prevention of process equipment, and information technology of process equipment. He received a Ph.D. degree in chemical process machinery from East China University of Science and Technology, China, in 1998. Between 2006-2007, he was a visiting scholar at University of Nottingham, UK.

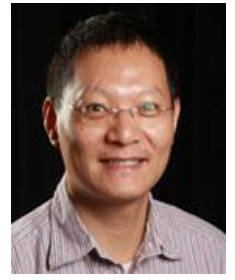

Hongbo Su is currently an assistant professor with Florida Atlantic University, Boca Raton, FL, USA. His primary research interests include quantitative remote sensing, surface hydrology and data assimilation. He received the Bachelor's degree in engineering from Wuhan University, Wuhan, China, in 1997, and the Ph.D. degree in cartography and geographic science from the Graduate School of Chinese Academy Sciences, Beijing, China, in 2002. He received another Ph.D. degree in surface hydrology from the Department of Civil and Environmental Engineering, Princeton University, Princeton, NJ, USA, in 2006. 